\title{
Assessment of Capacitor-based Charge Estimators for Piezoelectric Actuators
}

\author{
Mortezaa Mohammadzaheri \\ Department of Mechanical and \\ Industrial Engieering \\ Sultan Qaboos University \\ Muscat, Oman \\ 0000-0002-8187-6375 \\ Issam Bahadur \\ Department of Mechanical and \\ Industrial Engieering \\ Sultan Qaboos University \\ Muscat, Oman \\ 0000-0003-2373-6590
}

\author{
Sami AlSulti \\ Department of Mechanical and \\ Industrial Engieering \\ Sultan Qaboos University \\ Muscat, Oman \\ s.salti@squ.edu.om \\ Mohammadreza Emadi \\ Department of Mechanical and \\ Industrial Engieering \\ Sultan Qaboos University \\ Muscat, Oman \\ 0000-0002-1127-1311
}

\author{
Mojtaba Ghodsi \\ School of Energy \& Electronic \\ Engineering \\ University of Portsmouth \\ Portsmouth, UK \\ 0000-0002-8762-9498
}

\begin{abstract}
This paper focuses at charge estimators of piezoelectric actuators with a sensing capacitor. They have been claimed in the literature to be outperformed by their newly emerged competitors, charge estimators with a sensing resistor, widely known as digital charge estimators. This paper proposes a digital implementation of capacitor-based estimators and compares them with resistor-based ones both analytically and experimentally. Although, the sensing capacitors are normally bulkier than the sensing resistors used in newer resistor-based estimators; a resistor-based estimator needs to have a variable resistance to deal with different excitation frequencies satisfactorily; this is a major drawback which does not exist in capacitor-based estimators. Both capacitor-based and resistorbased estimators, if designed appropriately, are quite comparable in terms of voltage drop and range of measurable charge. This research concludes that capacitor-based estimators, with right design and implementation, can be still of wide use in nanopositioning.
\end{abstract}

Keywords-piezoelectric, charge, actuator, capacitor, nanopositioning

\section{INTRODUCTION}

Nanopositioning is a core aspect of nanotechnology, aiming at precise motion control at nanometre scale. Scanning probe microscopy [1] (e.g. atomic force microscopy [2]), robotic surgery [3], micro aerial vehicles [4], precise machining [5] and manipulation of miniature parts [6] and biological cells [7] are some application areas of nanopositioners. Different actuators have been used for nano/micropositioning e.g. worm gears [8], magnetostrictive actuators [9] and linear motors [10]. Amongst all, piezoelectric actuators are the least bulky and the most precise ones [11]. They are currently the most common actuators for nanopositioning and are likely to maintain this status for years [12-15].

The major variable in piezo-actuated (and other types of) nanopositioning is the actuator position, i.e. displacement of an unfixed point/surface of the actuator from its relaxing state [16]. Experiments have indicated that the charge of a piezoelectric actuator is proportional to its position for a wide area of operating [17-20]. That is, a charge estimator can replace an expensive and troublesome accurate position/displacement sensor; this motivates research on charge estimation of piezoelectric actuators $[11,21,22]$. This paper assesses a specific type of charge estimators for piezoelectric actuators and shows its merit.

\section{DifFERENT TyPES OF CHARGE ESTIMATORS OF PIEZOELECTRIC ACTUATORS}

Three prominent types of charge estimators have been invented for piezoelectric actuators. Prior to explanation of estimator types, it should be noted that the term "charge estimator" is not very common in the literature of piezoactuated nanopositioning; instead, terms "charge amplifier" $[17,18,23,24]$, "charge drive(r)" $[19,25,26]$ or "charge control system" [27-29] have been more widely used. Charge drivers/amplifiers normally include a charge estimator. However, some of charge estimators do not work independent of the entire charge control circuit; examples are the estimators of charge control systems with two switching current or voltage sources[30, 31]. All charge estimators which cannot work independent of charge amplifiers/drivers are outside the scope of this paper. On the other hand, most charge amplifiers or drivers consist of two separable subsets, a charge estimator and a feedback control system. In such drivers/amplifiers, separation of the controller and the estimator can further clarify the design idea and pave the way for improvements and new applications. Separable estimators can be also used merely in open loop. A review of charge drivers can be found in [26]; the focus of this paper is particularly on charge estimators. Here are main types of charge estimators for the piezoelectric actuators:

Type $i$ : charge estimators with a sensing capacitor and an initialisation circuit

Type ii: charge estimators with a sensing capacitor and a high pass filter

Type iii: charge estimators with a sensing resistor.

A. Type $i$-charge estimators with a sensing capacitor and an initialisation circuit

Fig. 1 is a simplified schematic of the charge estimator presented in the pioneer work of [27] (as a part of a charge control system). $V_{e}$ is the excitation voltage, the voltage applied to the actuator, and $V_{S}$ is the sensing voltage, the voltage across the sensing capacitor of $C_{S}$. $V_{S}$ is not applied on the actuator; thus, it is widely called the voltage drop.

With neglecting the initialisation circuit and the current going to the voltmeter to measure, $V_{S}$, in Laplace domain, is ideally presented by (1): 


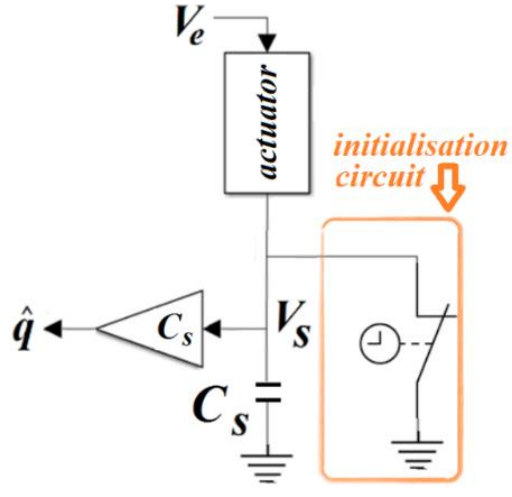

Fig. 1. Schematic of a charge estimator with a sensing capacitor and an initialisation circuit

$$
V_{S}=\frac{I_{P}}{C_{S} s}
$$

where $I_{P}$ is the current passing the piezoelectric actuator, in which $I_{P}=q s(2) . q$ is the charge applied on the piezoelectric actuator. (3) shows that the amplified $V_{S}$, passing through a voltage amplifier with a gain of $C_{S}$, can estimate the charge:

$$
V_{S}=\frac{q s}{C_{S} s}=\frac{q}{C_{S}} \Rightarrow \hat{q}=C_{S} V_{S} .
$$

However, $\hat{q}$, the estimated charge, may be unequal to $q$ due to impreciseness of (1). In reality, dielectric leakage of the piezoelectric actuator generates an additional low frequency (almost DC) small voltage [26]. This and other sources within the circuit result in an additional DC current, $I_{b}$, not presented in $(1-3) . I_{b}$ is added to $I_{P}$ and integrated by the sensing capacitor [32]. The effect of $I_{b}$ is presented in (4) and (5):

$$
V_{S}=\frac{I_{P}+I_{b}}{C_{S} s}=\frac{q}{C_{S}}+\frac{I_{b}}{C_{S} s} \Rightarrow q=C_{S} V_{S}-\frac{I_{b}}{s} .
$$

(3) and (4) result in $\hat{q}=q+\frac{I_{b}}{s}$.

$$
\begin{aligned}
& \text { the cause } \\
& \text { of drift }
\end{aligned}
$$

(4) and (5) show that, due to integration of $I_{b}$, non-existent in (1-3), the estimated charge, $\hat{q}$, ramps away the real charge of the piezoelectric actuator $q$. This phenomenon is called 'drift' and has been observed from the beginning of development of charge estimators for piezoelectric actuators [27, 28].

Initialisation circuits can remove drift to a very large extent; these circuits simply short circuit the sensing capacitor in sub-second periods of time [32] to interrupt integration of DC current. Figure 4 of [27] includes an initialisation circuit; in this circuit, a timer and a switch, as depicted in Fig.1, are directly involved in open-loop charge estimation. Figure 7 of [28] presents a more complicated initialisation circuit including current buffers. Both initialisation circuits reported in $[27,28]$ discharge the sensing capacitor every $400 \mathrm{~ms}$; that is, these estimators cannot capture any charge signal with a frequency lower than $5 \mathrm{~Hz}$ (two times the switching frequency [33]). In addition, all initialisation circuits have switches and suffer from problems associated with switch-based methods: (a) appearance of high-frequency parasitic voltages, and (b) limits imposed on operation frequency by switching speed [26].
B. Type iiA-charge estimators with a sensing capacitor and an analogue high pass filter

As an alternative to an initialisation circuit, a resistor of $R$ was used in parallel with the actuator [32], as depicted in Fig.2.

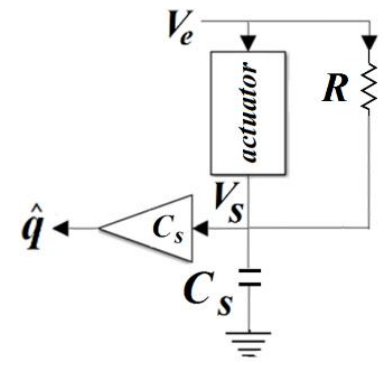

Fig. 2. Analogue implementation of a charge estimator with a sensing capacitor and high pass filter

A piezoelectric actuator roughly behaves like a capacitor, with a capacitance of $C_{P}$, from the electrical point of view [30]. The voltages across $R$ and across the actuator are same, as they are in parallel. Considering $I_{R}$ as the current passing $R$,

$$
I_{P} \frac{1}{C_{P} s}=I_{R} R .
$$

Considering (2) and (6), (7) presents $I_{S}$, the current entering the node with the voltage of $V_{S}$ (the cross junction in Fig.2):

$$
I_{S}=I_{P}+I_{R}=\left(1+\frac{1}{C_{P} R s}\right) I_{P}=\left(1+\frac{1}{C_{P} R s}\right) q s .
$$

Since $I_{S}$ (mostly) passes through $C_{S}$ :

$$
I_{S}=V_{S} C_{S} s
$$

(7) and (8) lead to (9):

$$
\hat{q}=V_{S} C_{S} \underbrace{\left(\frac{s}{s+\frac{1}{C_{P} R s}}\right)}_{\text {high pass filter }} .
$$

(9) has an additional high-pass filter compared to (3); this filter with a cut-off frequency of $\left(C_{P} R\right)^{-1} \mathrm{rad} / \mathrm{s}$ removes DC (or very low frequency) current of $I_{b}$. Disadvantageously, the filter suppresses other low frequency components as well; hence, this estimator may not capture low frequency charge signals.

\section{Type iii- charge estimators with a sensing resistor}

As to (1), the main role of the sensing capacitor is to integrate a current signal to estimate charge. Digital implementation provides the opportunity to perform integration within a digital processor rather than with a capacitor. Fig.3 depicts such a charge estimator, where the sensing capacitor is replaced by a sensing resistor, where A/D stands for analogue to digital converter. Voltage drop, $V_{S}$, still exists in this estimator; though, use of a resistor instead of a capacitor has been claimed to result in a smaller voltage drop [20]. With neglecting the tiny current going to A/D,

$$
V_{S}=I_{R S} R_{S}=I_{P} R_{S},
$$


where $I_{R S}$ stands for the current passing the sensing resistor of $R_{S .}$ (2) and (10) lead to (11):

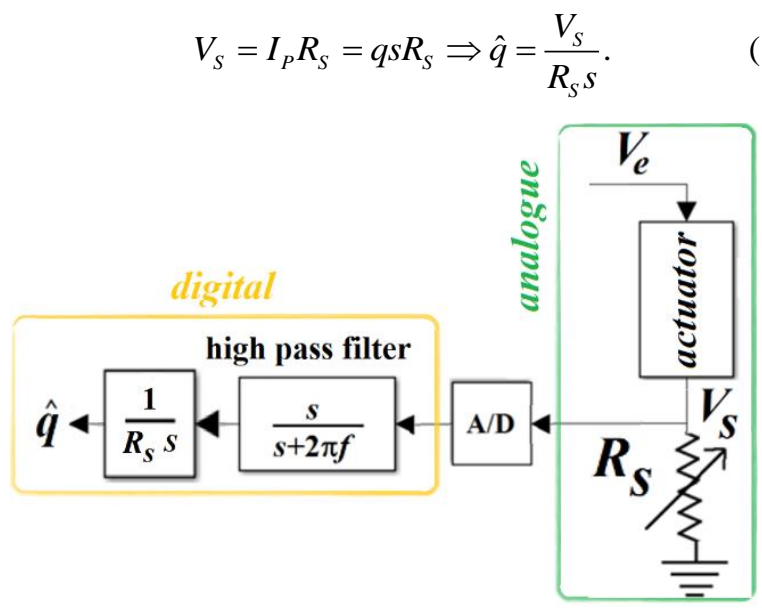

Fig. 3. A charge estimator with a sensing resistor

However, as mentioned in subsection II.B, dielectric leakage of the piezoelectric actuator generates an additional low frequency (nearly DC) small voltage[34]. This voltage together with A/D offset voltage form an additional DC voltage, $V_{b}$, not presented in (10 and 11) [35]. In other words, $V_{S}+V_{b}$, enters the digital processor rather than $V_{S}$. Therefore,

$$
\hat{q}=\frac{V_{S}+V_{b}}{R_{S} s}=\frac{V_{S}}{R_{S} s}+\frac{V_{b}}{R_{S} s} .
$$

That is, drift (integration of a minute DC signal over time) exists in charge estimators with a sensing resistor too. It is why there is a high pass filter to suppress low frequency components before integration.

Inasmuch as charge estimators with a sensing resistor (type iii) can be only implemented as digital systems, they are widely referred as 'digital' $[17,18,36,37]$. As another reason for the title of digital, only analogue implementation of type $i$ and ii estimators have been reported in the literature. In this paper, however, these estimators are referred to their sensing resistors, because charge estimators with a sensing capacitor can be also digitally implemented, as detailed in II.D.

\section{Type iiB-charge estimators with a sensing capacitor and a digital high pass filter}

This paper proposes the estimator of Fig. 4, an equivalent of Fig. 2 with digital implementation, where $f$ is the cutting frequency of the high pass filter in $\mathrm{Hz}$.

\section{Problem Statement}

As detailed in section II, amongst the three major types of charge estimators for piezoelectric actuators, type $\mathrm{i}$, including a switch or switches, witnesses high frequency disturbances, limited operating frequency and distortion of low frequency signals as detailed in subsection II.A. Types ii and iii present much more promising characteristics. However, they share two major drawbacks:

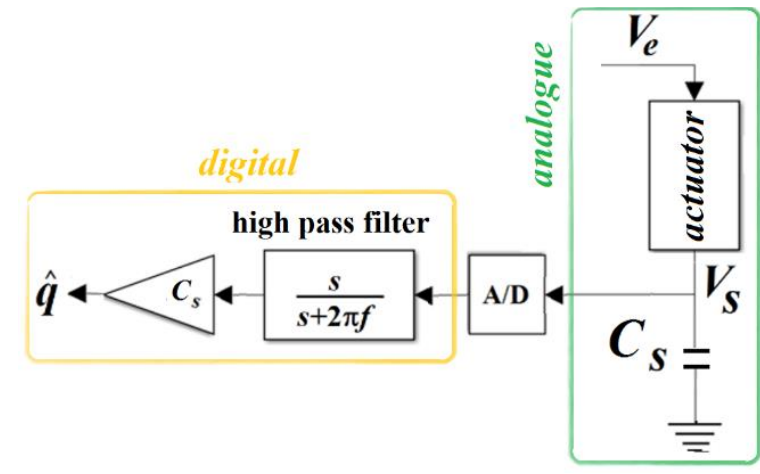

Fig. 4. Proposed digital implementation of a charge estimator with a sensing capacitor and a high pass filter

1. The high pass filter, avoiding drift, distorts low frequency charge signals,

2. The voltage across the sensing element, known as voltage drop, is not applied on the actuator and is practically wasted.

The first drawback can be appropriately tackled e.g. with use of the piezoelectric voltage signal, processed through an artificial neural network, similar to the method described in [36]. This method can be utilised for both type ii and iii charge estimators. Hence, this drawback will no longer be discussed in the paper.

The second drawback, voltage drop, is the remaining decisive matter. Experimental results presented in [18] demonstrate that a type iii estimator witnesses much less voltage drop compared to a type ii one, for an identical problem; therefore, it was concluded that type iii estimators outperform type ii ones in terms of voltage drop. This conclusion is re-visited and challenged in this paper. As a critical weak point of [18], the sensing elements (capacitor or resistor) of both type ii and type iii estimators were chosen intuitively; hence, no general conclusion could be drawn out of their comparison.

In order to compare type ii and iii charge estimators, the relationships of sensing resistance/capacitance with voltage drop and the range of estimated charge is analytically formulated. Then, both type ii and type iii estimators are designed so as to result in an (apt) voltage drop with the amplitude of $1 \mathrm{~V}$ for a cosinusoidal excitation voltage. Apt voltage drop, as defined in $[35,37,38]$, is the smallest voltage drop which can be used to estimate charge with no sacrifice in precision. The criteria for aptness of voltage drops have been detailed in $[35,37,38]$ and are not repeated here; it is just assumed that $1 \mathrm{~V}$ is the amplitude of apt (desired) voltage drop. The research question of this paper, answered through analytical and experimental investigations, is 'whether a type ii charge estimator can be designed to perform comparable to a type iii one, in realisation of the apt voltage drop?' A yes answer to this question defies the superiority of type iii estimators over type ii ones, a claimed in [18]. Type iiB estimators, with a digital filter, are used in this research to increase comparability with type iii estimators.

\section{APPROXIMATE ANALYTICAL FORMULATION}

This section analytically formulates the relationship of the sensing capacitance/resistance in a type ii/iii charge estimator and the voltage drop. The piezoelectric actuator is approximated by a capacitor, $C_{P}[18]$. 


\section{A. Analytical formulation for type iiB charge estimators}

In Fig. 4, the piezoelectric actuator and the sensing capacitor are in parallel; thus, their equivalent impedance, $Z$, and the current passing the actuator, $I_{P}$, can be found using (13 and 14):

$$
\begin{gathered}
Z(s)=\frac{1}{C_{P} s}+\frac{1}{C_{S} s}=\frac{C_{S}+C_{P}}{C_{S} C_{P} s} . \\
I_{P}(s)=V_{e}(s) \frac{C_{S} C_{P} s}{C_{S}+C_{P}} .
\end{gathered}
$$

Then, the voltage drop, $V_{S}$, can be calculated as the following:

$$
V_{S}(s)=\underbrace{V_{e}(s) \frac{C_{S} C_{P} s}{C_{S}+C_{P}}}_{I_{P}} \times \frac{1}{C_{S} s}=V_{e}(s) \frac{C_{P}}{C_{S}+C_{P}} .
$$

As a result of (15), $V_{S}$ is proportional to the excitation voltage, $V_{e}$; therefore, a bias in $V_{e}$ leads to a bias in $V_{S}$. In addition, considering $A_{e}$ and $A_{S}$ as the magnitudes of $V_{e}$ and $V_{S}$, respectively. (15) leads to (16):

$$
A_{S}=A_{e} \frac{C_{P}}{C_{S}+C_{P}} .
$$

Consequently, in order to achieve an $A_{S}$ of $1 \mathrm{~V}$, for a given $A_{e}$, the sensing capacitor should be chosen according to (17):

$$
C_{S}=C_{P}\left(1+A_{e}\right) \text {. }
$$

A cosinusoidal excitation voltage, leads to a cosinusoidal voltage drop as to (15). In this case, $q=C_{S} V_{S}=C_{S} A_{S} \cos \omega t$. As a result, $q_{\text {range-ii }}=2 C_{S}$.

For an $A_{S}$ of $1 \mathrm{~V},(17)$ and (18) lead to

$$
q_{\text {range-ii }}=2 C_{P}\left(1+A_{e}\right) .
$$

\section{B. Analytical formulation for type iii charge estimators}

For the system depicted in Fig.3, excluding the high pass filter, (20-22) substitute (13-15):

$$
\begin{gathered}
Z(s)=\frac{1}{C_{P} s}+R_{S}=\frac{1+R_{S} C_{P} s}{C_{P} s} . \\
(20) \\
I_{P}(s)=V_{e}(s) \frac{C_{P} s}{1+R_{S} C_{P} s} . \\
V_{S}(s)=\underbrace{V_{e}(s) \frac{C_{P} s}{1+R_{S} C_{P} s}}_{I_{P}} \times R_{S}=V_{e}(s) \frac{R_{S} C_{P} s}{1+R_{S} C_{P} s} \\
\Rightarrow \frac{V_{S}(s)}{V_{e}(s)}=\frac{R_{S} C_{P} s}{R_{S} C_{P} s+1} .
\end{gathered}
$$

For the approximate linear system presented by (22), a cosinusoidal excitation voltage without a bias, $V_{e}=A_{e} \cos \omega t$, leads to a sensing voltage of

$V_{S}=A_{S}\left(\cos \omega t+0.5 \pi-\tan ^{-1} R_{S} C_{P}\right)=A_{S}\left(\cos \omega t+0.5 \pi-\tan ^{-1} R_{S} C_{P}\right)$

$\simeq-A_{S}(\sin \omega t)$.

where the amplitude of $A_{S}$ and $A_{e}$ have the following relationship:

$$
\begin{aligned}
& \frac{A_{S}}{A_{e}}=\left|\frac{R_{S} C_{P} j \omega}{R_{S} C_{P} j \omega+1}\right|=\frac{R_{S} C_{P} \omega}{\sqrt{\left(R_{S} C_{P} \omega\right)^{2}+1}} \Rightarrow A_{S} \sqrt{\left(R_{S} C_{P} \omega\right)^{2}+1} \\
& =A_{e} R_{S} C_{P} \omega \Rightarrow A_{S}{ }^{2}\left(\left(R_{S} C_{P} \omega\right)^{2}+1\right)=A_{e}^{2}\left(R_{S} C_{P} \omega\right)^{2}
\end{aligned}
$$

Hence, $A_{S}=\frac{A_{e} R_{S} C_{P} \omega}{\sqrt{1+\left(R_{S} C_{P} \omega\right)^{2}}}$.

Consequently, in order to achieve an $A_{S}$ of $1 \mathrm{~V}$, for a given $A_{e}$, the sensing resistor should be chosen according to (25):

$$
R_{S}=\frac{1}{C_{P} \omega \sqrt{A_{e}^{2}-1}},
$$

In order to calculate charge, with use of (10):

$$
\begin{aligned}
& q(t)=\int_{o}^{t} I_{P} d \tau=\int_{o}^{t} \frac{V_{S}}{R_{S}} d \tau=\int_{o}^{t}-\frac{A_{S}}{R_{S}} \sin \omega \tau d \tau=-\left.\frac{1}{R_{S} \omega} \cos \omega \tau\right|_{0} ^{t} \\
& =-\frac{1}{R_{S} \omega}(\cos \omega t-1) .
\end{aligned}
$$

As a result, $q_{\text {range-iii }}=\frac{2}{R_{S} \omega}$.

In order to have an $A_{S}$ of $1 \mathrm{~V},(25$ and 26) lead to

$$
q_{\text {range-iii }}=2 C_{P} \sqrt{A_{e}^{2}-1} \sqcap 2 C_{P} A_{e} .
$$

Considering $A_{e} \square 1$ in most cases.

So far in subsection IV.B, bias was not considered. Now, let us assume a cosinusoidal excitation voltage with bias of $\mathrm{B}$, or $V_{e}=A_{e} \cos \omega t+\mathrm{B}$. In this case, as (22) is linear, the sensing voltage, $V_{S}$, can be assumed as sum of two components influenced by cosinusoidal and fixed (bias) excitations. The final value of the component of $V_{S}$, influenced by $\mathrm{B}, V_{S B}$ is shown to be zero in (28):

$$
V_{S B}(s)=\lim _{s \rightarrow 0} s \frac{R_{S} C_{P} s}{R_{S} C_{P} s+1} \frac{B}{s}=0 .
$$

That is, excitation bias has no enduring effect in type iii charge estimator of piezoelectric actuators.

\section{Results of Approximate Analytical Investigation}

Comparison of IV.A and IV.B can be summarised as following:

1) Theoretically, to achieve any apt (desired) voltage drop, with type ii/iii charge estimator a capacitor/ a resistor can be found according to (17)/(25).

2) The capacitor/resistor leading to the apt voltage drop in type ii/iii estimators, according. to $(17) /(25)$ is independent of /dependent on of excitation frequency. This is an advantage for type ii (capacitorbased) charge estimators.

3) Biased excitation voltage leads to proportionally biased/unbiased voltage drop (= sensing voltage) in type ii/iii estimators.

4) With the same voltage drop, type ii charge estimators can estimate slightly higher charges than type iii (resistor-based) ones, as to (19 and 27). 
These quadruple listed points do not support the claimed superiority of type iii estimators over type ii ones in the literature, and are mostly in favour of capacitor-based type ii estimators. Although, some practical factors such as large size of high capacitance capacitors, the outputs of (17), may be in favour of resistor-based estimators. The following sections experimentally compare type ii and type iii estimators.

\section{EXPERIMENTATION}

Fig.5 depicts the experimental setup, which is the implementation of Fig.4. $f$ in the high pass filter is $5 \mathrm{~Hz}$. The digital processor is a personal computer with Intel Core i72600 CPU @ 3.4GHz and 12 GB RAM equipped with MATLAB 9.1 /Simulink 8.8 software including Simulink Real-Time Desktop Toolbox 5.3. The actuator is a $7 \times 7 \times 42$ $\mathrm{mm}^{3}$ piezoelectric stack made by PiezoDrive Company [39]. The capacitance of the actuator, $C_{P}$, was found to be $6.23 \mu \mathrm{F}$ using an LCR meter at the amplitude of $1 \mathrm{~V}$ and the frequency of $1 \mathrm{kHz}$. The amplifier is an AETECHRON model 7114 liner power amplifier. A multifunctional card of National Instruments PCIe-6323 was employed as an Input-Output (I/O card) to connect the computer and analogue parts.

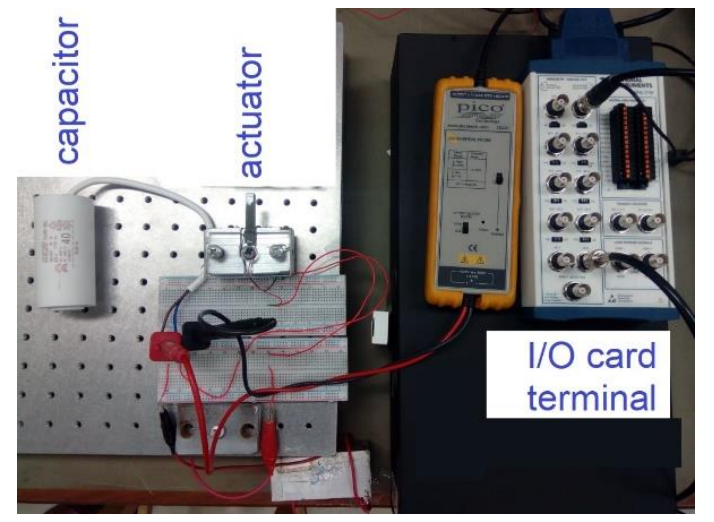

Fig. 5. Implementation of Fig 4, excluding the computer and the amplifier

Then, the excitation voltages of $V_{e}=A_{e} \cos \omega t$ were applied to the setup, where $f_{e}=\omega / 2 \pi(29)$. With the sensing capacitors of 20,40 and $80 \mu \mathrm{F}$ and their respective $A_{e}$ of $4.21,7.42$ and 13.84 $\mathrm{V} ; A_{S}$ and $q_{\text {range-ii }}$ were experimentally measured for the excitation frequency of $50 \mathrm{~Hz}$. Also, $A_{S}$ and $q_{\text {range-iii }}$ were measured on the implementation of Fig.3, with the sensing resistors of 37,67 and $121 \Omega$ and their respective $A_{e}$ of 4.21 , 7.42 and $13.84 \mathrm{~V}$, at the excitation frequency of $50 \mathrm{~Hz}$. The sampling frequency of $10 \mathrm{kHz}$ was used in experiments.

\section{RESULTS AND DISCUSSION}

According to section IV, for experiments detailed in section $\mathrm{V}$, it is theoretically expected that

$$
\begin{array}{ll}
\text { 1. } & A_{S}=1 \mathrm{~V} \\
\text { 2. } & q_{\text {range- } i i}=2 C_{S} \\
\text { 3. } & q_{\text {range-iii }}=2\left(C_{S^{-}} C_{P}\right)(30) .
\end{array}
$$

where (30) is a result of $(18,19$ and 27$)$. Table 1 presents the experimental results to assess the aforementioned theoretical expectations for the excitation frequency of $50 \mathrm{~Hz}$.

Table 1 shows that the estimated charge is in general higher than the one theoretically estimated; which is good news. Table 1 also demonstrates that, in the type ii estimator (with a sensing capacitor), experimental amplitudes of voltage drop $\left(A_{S}\right)$ are distinctively higher than their theoretically expected value of $1 \mathrm{~V}$. This partly agrees with claims of [18].

\begin{tabular}{|c|c|c|c|c|c|}
\hline \multicolumn{2}{|c|}{$A_{e}=13.84 \mathrm{~V}$} & \multicolumn{2}{|c|}{$A_{e}=7.42 \mathrm{~V}$} & \multicolumn{2}{|c|}{$A_{e}=4.21 \mathrm{~V}$} \\
\hline \multicolumn{2}{|c|}{ Type iiB,$C_{S}=80 \mu \mathrm{F}$} & \multicolumn{2}{|c|}{ Type iiB, $C_{S}=40 \mu \mathrm{F}$} & \multicolumn{2}{|c|}{ Type iiB, $C_{S}=20 \mu \mathrm{F}$} \\
\hline$A_{S}$ & $q_{\text {range- } i i}$ & $A_{S}$ & $q_{\text {range }-i i}$ & $A_{S}$ & $q_{\text {range-ii }}$ \\
\hline $1.20 \mathrm{~V}$ & $201.04 \mu \mathrm{C}$ & $1.16 \mathrm{~V}$ & $89.60 \mu \mathrm{C}$ & 1.07 & $42.69 \mu \mathrm{C}$ \\
\hline \multicolumn{2}{|c|}{ Type iii with $R_{S}=37 \Omega$} & \multicolumn{2}{|c|}{ Type iii with $R_{S}=69 \Omega$} & \multicolumn{2}{|c|}{ Type iii with $R_{S}=121 \Omega$} \\
\hline$A_{S}$ & $q_{\text {range }-i i i}$ & $\boldsymbol{A}_{S}$ & $q_{\text {range -iii }}$ & $A_{S}$ & $q_{\text {range -iii }}$ \\
\hline $1.03 \mathrm{~V}$ & $183.40 \mu \mathrm{C}$ & $0.98 \mathrm{~V}$ & $93.00 \mu \mathrm{C}$ & $0.98 \mathrm{~V}$ & $49.83 \mu \mathrm{C}$ \\
\hline
\end{tabular}

TABLE I. EXPERIMENTAL RESULTS FOR THE EXCITATION FREQUENCY OF $50 \mathrm{~Hz}$

Although, the discrepancy of experimental and theoretical $A_{S}$ is not significant, it can lead to saturation. That is, for an A/D input range of e.g. $[-1+1] \mathrm{V}, V_{S}$ exceeds the range at times and is saturated down into A/D input range; the correct charge will be unavailable in such occasions. The alternative is to use a higher sensing capacitance, $C_{S}$, than the one suggested by (17). According to Table 1, the issue of saturation exists in type iii estimators too, to a smaller extent; hence, sensing resistors smaller than the ones suggested by (25) may be alternatively used.

In general, type ii estimators (with a sensing capacitor) have a major advantage over type iii ones, independence of operating frequency, and a major disadvantage, the bulkiness of capacitors. In terms of voltage drop and charge, they are well comparable, and there are ways to systematically deal with the saturation problem, e.g. the methods presented for type iii estimators in $[35,40]$.

\section{CONCLUSION}

This paper compares the most recent types of charge estimators for piezoelectric actuators, type ii, the estimators with a sensing capacitor and a high pass filter, and type iii, the estimators with a sensing resistor. In order to improve comparability, the digital version of type ii estimators was proposed and implemented, depicted in Fig.4 of the paper. In the literature, it has been claimed that type ii estimators witness much higher voltage drop than type iii ones, in similar operating conditions. On this basis, it has been concluded that type iii estimators outperform type ii ones. This conclusion was challenged thorough analytical and experimental comparison.

Based on analytical formulation, specific values of the sensing capacitance/resistance were calculated to result in a voltage drop with the amplitude of $1 \mathrm{~V}$. The calculated sensing capacitors (of type ii estimators) are bulkier than the sensing resistors (of type iii estimators). In experiments, type ii estimators showed higher amplitude of voltage drop than $1 \mathrm{~V}$ by $16 \%$ and $20 \%$; while, type iii estimators result in voltage drop amplitudes much closer $1 \mathrm{~V}$. However, use of higher capacitances than calculated ones can dim this drawback. On the other hand, it was shown that analytically calculated sensing capacitances (in type ii estimators) are independent of excitation frequency, unlike the sensing resistances of type iii estimators. In total, it can be concluded type ii estimators, if designed and implemented appropriately, can be still of wide use in nanopositioning.

\section{REFERENCES}

[1] L. Zhang, X. Chen, J. Huang, H. Li, L. Chen, and Q. J. R. o. S. I. Huang, "A method to correct hysteresis of scanning probe microscope images based on a sinusoidal model," vol. 90, p. 023704, 2019.

[2] Y. H. Teh, "Labview Based Pid Algorithm Development for Z Motion Control in Atomic Force Microscopy," UTAR, 2015. 
[3] S. Saedi, A. Mirbagheri, A. Jafari, and F. Farahmand, "A local hybrid actuator for robotic surgery instruments," International Journal of Biomechatronics and Biomedical Robotics 33, vol. 3, pp. 100-105, 2014.

[4] J. Li, H. Huang, T. J. S. Morita, and A. A. Physical, "Stepping piezoelectric actuators with large working stroke for nano-positioning systems: a review," vol. 292, pp. 39-51, 2019.

[5] W. Xu, Y. J. M. S. Wu, and S. Processing, "Piezoelectric actuator for machining on macro-to-micro cylindrical components by a precision rotary motion control," vol. 114, pp. 439-447, 2019.

[6] H. Mehrabi, M. Hamedi, and I. J. M. T. Aminzahed, "A novel design and fabrication of a micro-gripper for manipulation of micro-scale parts actuated by a bending piezoelectric," pp. 1-9, 2019.

[7] X. Li and C. C. Cheah, "Robotic cell manipulation using optical tweezers with unknown trapping stiffness and limited fov," IEEE/ASME Transactions on Mechatronics, vol. 20, pp. 1624-1632, 2015.

[8] V. Protopopov, "Beam Alignment and Positioning Techniques," in Practical Opto-Electronics, ed: Springer, 2014, pp. 309-334.

[9] M. Ghodsi, A. Saleem, A. Özer, I. Bahadur, K. Alam, A. Al-Yahmadi, et al., "Elimination of thermal instability in precise positioning of Galfenol actuators," in Behavior and Mechanics of Multifunctional Materials and Composites 2016, 2016, p. 980008.

[10] L. Díaz Pérez, M. Torralba Gracia, J. Albajez García, and J. Yagüe Fabra, "One-Dimensional Control System for a Linear Motor of a TwoDimensional Nanopositioning Stage Using Commercial Control Hardware," Micromachines, vol. 9, p. 421, 2018.

[11] M. Mohammadzaheri and A. AlQallaf, "Nanopositioning systems with piezoelectric actuators, current state and future perspective," Science of Advanced Materials, vol. 9, pp. 1071-1080, 2017.

[12] A. E. ALamir, "Optimal control and design of composite laminated piezoelectric plates," Smart Structures and Systems, vol. 15, pp. 11771202, 2015

[13] D. Huber, M. Krommer, and H. Irschik, "Dynamic displacement tracking of a one-storey frame structure using patch actuator networks: Analytical plate solution and FE validation," Smart Structures and Systems, vol. 5, pp. 613-632, 2009.

[14] S. O. R. Moheimani, "Invited Review Article: Accurate and fast nanopositioning with piezoelectric tube scanners: Emerging trends and future challenges," Review of Scientific Instruments, vol. 79, Jul 2008.

[15] H. Xiang and Z. Shi, "Static analysis of a multilayer piezoelectric actuator with bonding layers and electrodes," Smart Structures and Systems, vol. 5, pp. 547-564, 2009.

[16] N. Miri, M. Mohammadzaheri, and L. Chen, "An enhanced physicsbased model to estimate the displacement of piezoelectric actuators," Journal of Intelligent Material Systems and Structures, p. 1045389X14546648, 2014.

[17] M. Bazghaleh, S. Grainger, B. Cazzolato, and T.-f. Lu, "An innovative digital charge amplifier to reduce hysteresis in piezoelectric actuators," presented at the Australian Robotics and Automation Association (ACRA), Brisbane, Australia, 2010.

[18] M. Bazghaleh, S. Grainger, M. Mohammadzaheri, B. Cazzolato, and T. Lu, "A digital charge amplifier for hysteresis elimination in piezoelectric actuators," Smart Materials and Structures, vol. 22, p. 075016, 2013.

[19] J. Minase, T. F. Lu, B. Cazzolato, and S. Grainger, "A review, supported by experimental results, of voltage, charge and capacitor insertion method for driving piezoelectric actuators," Precision Engineering, vol. 34, pp. 692-700, 2010.

[20] K. A. Yi and R. J. Veillette, "A charge controller for linear operation of a piezoelectric stack actuator," IEEE transactions on control systems technology, vol. 13, pp. 517-526, 2005.

[21] C. Yang, C. Li, and J. Zhao, "A nonlinear charge controller with tunable precision for highly linear operation of piezoelectric stack actuators," IEEE Transactions on Industrial Electronics, vol. 64, pp. 8618-8625, 2017.

[22] S.-T. Liu, J.-Y. Yen, and F.-C. Wang, "Compensation for the residual error of the voltage drive of the charge control of a piezoelectric actuator," Journal of Dynamic Systems, Measurement, and Control, vol. 140, pp. 1-9, 2018.

[23] A. J. Fleming and S. O. R. Moheimani, "A grounded-load charge amplifier for reducing hysteresis in piezoelectric tube scanners," Review of Scientific Instruments, vol. 76, pp. 0737071-5, 2005.

[24] V. S. C. Chillara, A. K. Ramanathan, and M. J. Dapino, "Self-sensing piezoelectric bistable laminates for morphing structures," Smart Materials and Structures, 2020.

[25] S. Rios and A. Fleming, "Control of Piezoelectric Benders Using a Charge Drive," in Proc. Actuator, 2014.

[26] M. Bazghaleh, S. Grainger, and M. Mohammadzaheri, "A review of charge methods for driving piezoelectric actuators," Journal of Intelligent Material Systems and Structures, vol. 29, pp. 2096-2104, 2018.

[27] R. H. Comstock, "Charge control of piezoelectric actuators to reduce hysteresis effects," ed: Google Patents, 1981.

[28] J. A. Main, E. Garcia, and D. V. Newton, "Precision position control of piezoelectric actuators using charge feedback," Journal of Guidance, control, and dynamics, vol. 18, pp. 1068-1073, 1995.

[29] T. Jin, Y. Peng, Z. Xing, and L. Lei, "A charge controller for synchronous linear operation of multiple piezoelectric actuators," IEEE Access, vol. 7, pp. 90741-90749, 2019.

[30] C. V. Newcomb and I. Flinn, "Improving the linearity of piezoelectric ceramic actuators," Electronics Letters, vol. 18, pp. 442-444, 1982.

[31] L. Huang, Y. T. Ma, Z. H. Feng, and F. R. J. R. o. S. I. Kong, "Switched capacitor charge pump reduces hysteresis of piezoelectric actuators over a large frequency range," vol. 81, p. 094701, 2010.

[32] A. J. Fleming and S. Moheimani, "Improved current and charge amplifiers for driving piezoelectric loads, and issues in signal processing design for synthesis of shunt damping circuits," Journal of Intelligent Material Systems and Structures, vol. 15, pp. 77-92, 2004.

[33] I. D. Landau and G. Zito, Digital Control Systems: Springer London, 2006.

[34] M. Bazghaleh, M. Mohammadzaheri, S. Grainger, B. Cazzolato, and T. F. Lu, "A new hybrid method for sensorless control of piezoelectric actuators," Sensors and Actuators A: Physical, vol. 194, pp. 25-30, 2013.

[35] M. Mohammadzaheri, M. Emadi, M. Ghodsi, I. M. Bahadur, M. Zarog, and A. Saleem, "Development of a Charge Estimator for Piezoelectric Actuators: A Radial Basis Function Approach," International Journal of Artificial Intelligence and Machine Learning (IJAIML), vol. 10, pp. 3144, 2020.

[36] M. Bazghaleh, S. Grainger, M. Mohammadzaheri, B. Cazzolato, and T.F. Lu, "A novel digital charge-based displacement estimator for sensorless control of a grounded-load piezoelectric tube actuator," Sensors and Actuators A: Physical, 2013.

[37] M. Mohammadzaheri, M. Emadi, M. Ghodsi, E. Jamshidi, I. Bahadur, A. Saleem, et al., "A variable-resistance digital charge estimator for piezoelectric actuators: An alternative to maximise accuracy and curb voltage drop," Journal of Intelligent Material Systems and Structures, vol. 30, pp. 1699-1705, 2019.

[38] M. Mohammadzaheri;, M. Emadi;, H. Ziaiefar;, M. Ghodsi;, I. Bahadur;, M. Zarog;, et al., "Adaptive Charge Estimation of Piezoelectric Actuators, a Radial Basis Function Approach," presented at the 20th International Conference on Research and Education in Mechatronics Wels, Austria 2019.

[39] PiezoDrive. Piezoelectric actuators. Available: http://piezodrive.com/actuators.html

[40] M. Mohammadzaheri, H. Ziaeifar, I. Bahadur, M. Zarog, M. Emadi, and M. Ghodsi, "Data-driven Modelling of Engineering Systems with Small Data, a Comparative Study of Artificial Intelligence Techniques," in 2019 5th Iranian Conference on Signal Processing and Intelligent Systems (ICSPIS), 2019, pp. 1-5. 